\title{
Associação dos fatores sociodemográficos e patológicos com os resultados perinatais de gestantes adolescentes no sul do Brasil
}

Association of sociodemographic and pathological factors with perinatal outcomes of pregnant women in southern Brazil

\author{
G. R. Valoni ${ }^{1}$; R. S. Ribeiro'; S. M. Pelloso ${ }^{2}$; M. B. Antunes ${ }^{1 *}$ \\ ${ }^{I}$ Departamento de Medicina, Centro Universitário de Maringá (UNICESUMAR), 87050-900, Maringá-PR, Brasil. \\ ${ }^{2}$ Departamento de Ciências da Saúde, Universidade Estadual de Maringá (UEM), 87020-900, Maringá-PR, Brasil. \\ *bena77i@gmail.com
}

(Recebido em 07 de setembro de 2019; aceito em 30 de junho de 2020)

\begin{abstract}
Objetivou-se analisar a associação dos fatores sociodemográficos e patológicos com os resultados perinatais em gestantes adolescentes entre 12 a 18 anos, classificadas como alto risco e acompanhadas por um serviço especializado. Trata-se de um estudo exploratório, descritivo de abordagem quantitativa. Foram analisados 920 prontuários de gestantes de alto risco, no período de setembro de 2012 a setembro de 2013. Os dados foram submetidos aos testes qui-quadrado $\left(\mathrm{x}^{2}\right)$ e teste exato de Fisher. Do total, 6,7\% eram gestantes adolescentes, das quais $5,2 \%$ foram selecionadas para esse estudo por realizarem o parto no hospital referência. A média de idade foi de 16,7anos e em relação ao número de gestações $18,8 \%$ adolescentes já estavam na segunda gestação, predominou-se o parto normal com $52,1 \%$. Em relação às condições de riscos, nas condições clínicas pré-existentes, destaca-se a prevalência de hipertensão arterial $(8,3 \%)$ e dependência de drogas lícitas $(8,3 \%)$, nos antecedentes obstétricos o parto prematuro apresentou prevalência de 8,3\%. Já nas intercorrências na atual gestação, destacou-se a malformação fetal $(16,7 \%)$, seguido do trabalho de parto prematuro $(12,5 \%)$. Quando analisado o desfecho perinatal das adolescentes, aquelas que apresentaram condições clínicas pré-existentes obtiveram associação estatística significativa para baixo peso ano nascer $(p=0,03)$, intercorrência clínica na atual gestação apresentaram associação com o parto pré-termo $(p=0,04)$ e baixo peso ao nascer $(\mathrm{p}=0,001)$. Conclui-se que a gestação na adolescência se relaciona com os riscos maternos tendo como consequência as complicações neonatais, destacando o parto pré-termo e o baixo peso ao nascer.

Palavras-chave: Saúde Materno-Infantil, Complicações na Gravidez, Gravidez na Adolescência.
\end{abstract}

The objective of this study was to analyze the association of sociodemographic and pathological factors with perinatal outcomes in pregnant women aged 12 to 18 years, classified as high risk and accompanied by a specialized service. This is an exploratory, descriptive study of quantitative approach. We analyzed 920 medical records of high-risk pregnant women from September 2012 to September 2013. Data were submitted to chi-square $\left(\mathrm{x}^{2}\right)$ and Fisher's exact tests. Of the total, 6.7\% were adolescent pregnant women, of which $5.2 \%$ were selected for this study because they delivered at the referral hospital. The average age was 16.7 years and in relation to the number of pregnancies $18.8 \%$ adolescents were already in the second pregnancy, with normal birth with $52.1 \%$. Regarding the risk conditions, in the pre-existing clinical conditions, the prevalence of arterial hypertension (8.3\%) and dependence on licit drugs (8.3\%) stands out. In the obstetric antecedents, premature birth presented a prevalence of $8 \%$. , 3\%. In the complications during the current pregnancy, fetal malformation $(16.7 \%)$ was highlighted, followed by premature birth work $(12.5 \%)$. When analyzing the perinatal outcome of the adolescents, those who had pre-existing clinical conditions obtained a significant statistical association for low birth weight $(\mathrm{p}=0.03)$, clinical complications in the current pregnancy were associated with preterm delivery $(\mathrm{p}=0,04)$ and low birth weight $(\mathrm{p}=0.001)$. It is concluded that teenage pregnancy is related to maternal risks resulting in neonatal complications, highlighting preterm birth and low birth weight.

Keywords:Maternal and Child Health, Pregnancy Complications, Pregnancy in Adolescence.

\section{INTRODUÇÃO}

A gravidez na adolescência é um fenômeno mundial e, apesar da redução de seus índices, continua a ser um dos principais problemas de saúde pública e social em diversos países do mundo, inclusive no Brasil, devido aos resultados que podem ocasionar tanto para o recém-nascido, para a 
adolescente e à família [1, 2]. A Organização Mundial da Saúde (OMS) destaca que a gestação nesta fase é um dos principais fatores que contribui para a mortalidade materna e infantil, tendo como causas frequentes as complicações durante a gravidez e o parto. Estima-se que haja 16 milhões de adolescentes grávidas entre 15 e 19 anos e cerca de 2,5 milhões de meninas com menos de 16 anos dão à luz a cada ano nas regiões em desenvolvimento [3].

No Brasil, a estimativa é de que um em cada quatro nascidos seja proveniente de gravidez na adolescência [4]. Embora o Brasil apresente queda de 17\% em gestação na adolescência, segundo dados do Sistema de Informação sobre Nascidos Vivos (SINASC 2004-2015), na região Sul, o número de nascidos vivos de mulheres de 10 a 19 anos foi de 62.475 (11\%) [5].

Os resultados perinatais que ocorrem entre as gestantes adolescentes têm sido destacados por alguns estudos. $\mathrm{O}$ baixo peso ao nascer, por exemplo, tem sido associado ao número insuficiente de consultas de pré-natal, à primigestação e à prematuridade, que geralmente ocorrem em gestações adolescentes e adultas jovens [6]. O número de cesáreas também tem sido observado por alguns autores como expressivos entre gestantes adolescentes, fato que pode comprometer o futuro reprodutivo destas com a realização de sucessivas cesarianas nas gestações posteriores [7].

Além desses, a gravidez na adolescência também tem sido relacionada a outros desfechos perinatais, tais como: prematuridade, baixo peso ao nascer, valores reduzidos de Apgar no $5^{\circ}$ minuto de vida, deficiência de micronutrientes e restrição de crescimento intrauterino $[8,9]$.

Diante dos dados apresentados pela literatura, e a escassez de dados relacionados a gestação na adolescência associada a riscos gestacionais como condições clínicas pré-existentes, antecedentes obstétricos e intercorrências clínicas na atual gestação, o objetivo desse estudo foi analisar a associação dos fatores sociodemográficos e patológicos com os resultados perinatais em gestantes adolescentes entre 12 a 18 anos, classificadas como alto risco e acompanhadas por um serviço especializado.

\section{MATERIAL E MÉTODOS}

Trata-se de um estudo exploratório, retrospectivo, descritivo de abordagem quantitativa. Foram analisados 920 prontuários de gestantes classificadas como alto risco e acompanhadas por um serviço especializado na atenção secundária pelo Sistema Único de Saúde (SUS) no período de setembro de 2012 a setembro de 2013, com crianças nascidas em 2013-2014, para obtenção dos dados das crianças provenientes das gestantes acompanhadas.

O ambulatório especializado é vinculado a um hospital filantrópico referência para o atendimento às gestantes de alto risco de 26 municípios pertencentes a $15^{\mathrm{a}}$ Regional de Saúde do Paraná. A coleta de dados foi realizada por meio de consulta aos prontuários eletrônicos e físicos e pela carteirinha da gestante, no período entre novembro de 2016 e outubro de 2017.

Vale destacar que a gestação na adolescência segundo a Linha Guia da Rede Mãe Paranaense (2018), é considerada como risco intermediário e a partir da associação da idade biológica com as condições clínicas pré-existentes, antecedentes obstétricos e/ou intercorrências clínicas na atual gestação, considera-se a gestação como alto risco [10].

Foram selecionadas como variáveis independentes para compor o estudo aquelas relacionadas com as características sociodemográficas (idade, estado civil, escolaridade e cor), tipo de parto, número de consultas especializadas e de gestações e características obstétricas (riscos relacionados a condições clínicas pré-existentes, antecedentes obstétricos e intercorrências na atual gestação) e as variáveis dependentes relacionadas ao desfecho perinatal: baixo peso ao nascer (BPN) (RN com peso $<2.500 \mathrm{~g}$ ), Apgar no $5^{\circ}$ minuto (menores que 7) pré-termo (nascidos antes de 37 semanas), Parto Normal e Parto Cesárea.

Os critérios de inclusão foram: gestantes adolescentes (idade entre 12 e 18 anos, conforme o Estatuto da Criança e Adolescente (ECA)), classificadas como alto risco pela Atenção Primária à Saúde (APS), ter realizado o parto no hospital de referência, ter comparecido a pelo menos uma consulta no ambulatório especializado. Os critérios de exclusão se referem ao não atendimento aos critérios anteriormente descritos. As informações foram registradas em um formulário previamente elaborado, denominado Gestantes de Alto Risco (GAR), e inserido em planilha eletrônica da Microsoft Corporation Redmond (WA, USA). 
A análise estatística foi realizada com auxílio do Software Epi Info versão 3.5.1, programa de domínio público criado pelo Centers for Disease Control and Prevention (CDC). Os dados foram submetidos aos testes Qui-quadrado $\left(\mathrm{x}^{2}\right)$ e Teste Exato de Fisher. Todas as discussões foram realizadas em nível de 5\% de significância $(\mathrm{p}<0,05)$ e intervalo de confiança de $95 \%$.

Para o desenvolvimento deste estudo foram respeitados todos os aspectos éticos nacionais e internacionais de pesquisa envolvendo seres humanos contidos na Resolução $n^{\circ}$ 466/12 do CNS/MS, sendo aprovado pelo parecer $n^{\circ} 2.287 .476$.

\section{RESULTADOS}

Do total de 920 registros que constituiu a população em estudo, $62(6,74 \%)$ eram gestantes adolescentes, das quais $48(5,22 \%)$ foram selecionadas por atender os critérios de inclusão e exclusão estabelecidos (Figura 1).

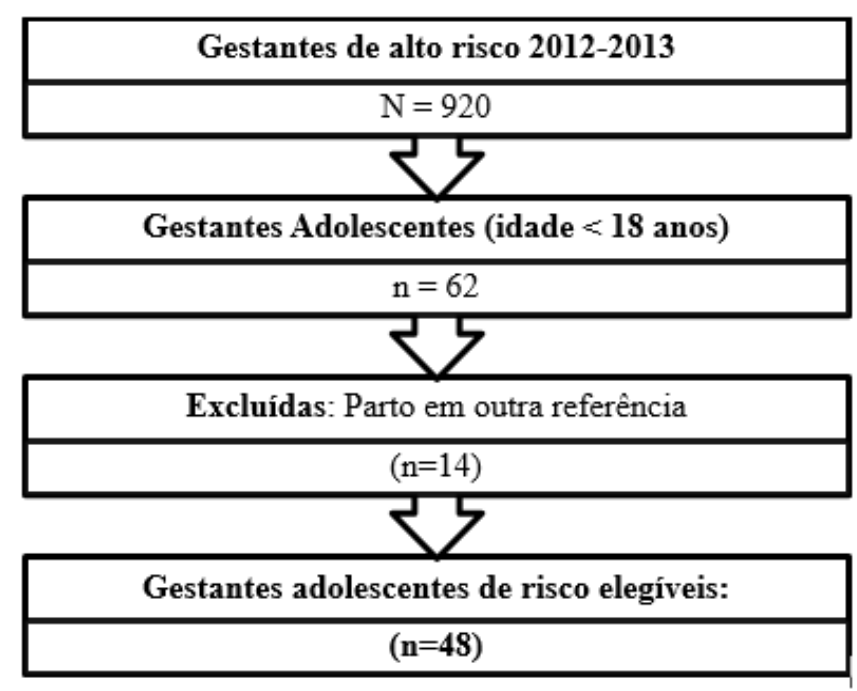

Figura 1: Elegibilidade da amostra referente às gestantes adolecentes. Fonte: os autores.

Em relação às características apresentadas, a média de idade foi de $16,70( \pm 1,39)$ anos (idade mínima de 12 e máxima de 18 anos), 70,83\% (34) declararam-se ser da cor branca e 79,17\% (38) das adolescentes não viviam com o companheiro. Em relação à escolaridade, 58,33\% (28) estavam cursando o ensino médio. Quanto ao número de consultas de pré-natal no ambulatório de risco, $52,08 \%$ (25) apresentaram de uma a três consultas especializadas e apenas 12,50\% (06) realizaram sete ou mais consultas. Em relação ao número de gestações 18,75\% (09) adolescentes já estavam na segunda gestação. Quanto ao tipo de parto, predominou-se o parto normal com 52,08\% (25), entretanto, vale destacar que dois foram contados em parto de gemelares e foram excluídos dois por óbito fetal (Tabela 1). 
Tabela 1: Características das gestantes adolescentes classificadas como alto risco e acompanhadas em ambulatório especializado em Maringá, PR, Brasil.

\begin{tabular}{|c|c|c|}
\hline Variáveis & $\mathrm{n}=48$ & $\%$ \\
\hline \multicolumn{3}{|l|}{ Estado civil } \\
\hline Vive com o companheiro & 10 & 20,83 \\
\hline Não vive com o companheiro & 38 & 79,17 \\
\hline \multicolumn{3}{|l|}{ Escolaridade } \\
\hline Não Alfabetizada & 01 & 2,09 \\
\hline Ensino Fundamental & 19 & 39,58 \\
\hline Ensino Médio & 28 & 58,33 \\
\hline \multicolumn{3}{|l|}{ Cor } \\
\hline Branca & 34 & 70,83 \\
\hline Preta & 03 & 6,25 \\
\hline Parda & 11 & 22,92 \\
\hline \multicolumn{3}{|l|}{ Número de Consultas* } \\
\hline $1-3$ & 25 & 52,08 \\
\hline $4-6$ & 17 & 35,42 \\
\hline$\geq 7$ & 06 & 12,50 \\
\hline \multicolumn{3}{|l|}{ Número de Gestações } \\
\hline 1 & 39 & 81,25 \\
\hline 2 & 09 & 18,75 \\
\hline \multicolumn{3}{|l|}{ Tipo de Parto** } \\
\hline Normal*** & 25 & 52,08 \\
\hline Cesárea & 23 & 47,92 \\
\hline
\end{tabular}

Em relação às condições de riscos apresentados pelas gestantes adolescentes, nas condições clínicas pré-existentes destaca-se a prevalência de hipertensão arterial e dependência de drogas lícitas, ambas com 8,33\% (04). Nos riscos referentes a antecedentes obstétricos o parto prematuro também apresentou prevalência de 8,33\% (04). Já nas intercorrências na atual gestação, o risco prevalente foi à má formação fetal confirmada intra útero com prevalência de 16,67\% (08) seguido do trabalho de parto prematuro com 12,50\% (06) dos casos (Tabela 2). 
Tabela 2: Condições de risco das gestantes adolescentes acompanhadas no ambulatório especializado em Maringá, PR, Brasil.

\begin{tabular}{|c|c|c|}
\hline Variáveis & $\mathrm{n}(48)$ & $\%$ \\
\hline \multicolumn{3}{|c|}{ Condições Clínicas Pré-existentes* $(n=22)$} \\
\hline Hipertensão arterial & 04 & 8,33 \\
\hline Cardiopatias & 02 & 4,17 \\
\hline Asma & 02 & 4,17 \\
\hline Diabetes Mellitus & 02 & 4,17 \\
\hline Talassemia & 01 & 2,08 \\
\hline Toxoplasmose & 01 & 2,08 \\
\hline Epilepsia & 03 & 6,25 \\
\hline Neoplasias & 01 & 2,08 \\
\hline Obesidade Mórbida & 01 & 2,08 \\
\hline Psicose e depressão & 01 & 2,08 \\
\hline Dependência de drogas lícitas & 04 & 8,33 \\
\hline \multicolumn{3}{|l|}{ Antecedentes Obstétricos* $(n=7)$} \\
\hline Mortes fetais e neonatais & 01 & 2,08 \\
\hline Baixo peso ao nascer & 02 & 4,17 \\
\hline Parto prematuro & 04 & 8,33 \\
\hline \multicolumn{3}{|c|}{ Intercorrências na atual gestação* $(n=22)$} \\
\hline ITU de repetição e/ou complicada & 03 & 6,25 \\
\hline Retardo do Crescimento Intrauterino & 03 & 6,25 \\
\hline Trabalho de parto prematuro & 06 & 12,50 \\
\hline Má formação fetal confirmada & 08 & 16,67 \\
\hline Gemelaridade & 02 & 4,17 \\
\hline
\end{tabular}

* Existem gestantes que apresentaram mais de um risco.

A análise dos desfechos perinatais das gestantes adolescentes de acordo com as variáveis sociodemográficas está apresentada na Tabela 3. Gestantes que frequentaram sete ou mais consultas apresentaram $100 \%$ de parto pré-termo. 
Tabela 3: Desfecho perinatal de gestantes adolescentes de alto risco segundo variáveis sociodemográficas em Maringá, PR, Brasil.

\begin{tabular}{|c|c|c|c|c|c|c|c|c|c|c|}
\hline Variáveis & $\begin{array}{l}\text { Pré- } \\
\text { termo } \\
\mathrm{n}(\%)\end{array}$ & $p$ & $\begin{array}{c}\text { Baixo peso } \\
\text { ao nascer } \\
\mathrm{n}(\%)\end{array}$ & $p$ & $\begin{array}{c}\text { Apgar } 5^{\circ} \\
\text { minuto }(<7) \\
n(\%)\end{array}$ & $p$ & $\begin{array}{c}\text { Parto } \\
\text { Normal } \\
\mathrm{n}(\%)\end{array}$ & $p$ & $\begin{array}{c}\text { Parto } \\
\text { Cesárea } \\
\mathrm{n}(\%)\end{array}$ & $p$ \\
\hline Estado civil & & 0,52 & & 0,59 & & 0,12 & & 0,41 & & 0,41 \\
\hline Casada & $5(50,0)$ & & $6(60,0)$ & & $10(100,0)$ & & $6(60,0)$ & & $4(40,0)$ & \\
\hline Solteira & $21(55,3)$ & & $22(57,9)$ & & $30(78,9)$ & & $19(50,0)$ & & $19(50,0)$ & \\
\hline Escolaridade & & 0,39 & & 0,35 & & 0,85 & & 0,57 & & 0,41 \\
\hline $\begin{array}{l}\text { Não } \\
\text { Analfabetizada }\end{array}$ & - & & $1(100,0)$ & & $1(100,0)$ & & - & & $4(40,0)$ & \\
\hline $\begin{array}{l}\text { Ensino } \\
\text { Fundamental }\end{array}$ & $13(50,0)$ & & $13(50,0)$ & & $22(84,6)$ & & $14(53,8)$ & & $19(50,0)$ & \\
\hline Ensino Médio & $13(61,9)$ & & $14(66,7)$ & & $17(81,0)$ & & $11(52,4)$ & & $23(47,9)$ & \\
\hline Cor & & 0,38 & & 0,35 & & 0,07 & & 0,20 & & 0,20 \\
\hline Branca & $20(58,8)$ & & $22(64,7)$ & & $31(91,2)$ & & $16(47,1)$ & & $18(52,9)$ & \\
\hline Preta & $2(66,7)$ & & $1(33,3)$ & & $2(66,7)$ & & $3(100,0)$ & & - & \\
\hline Parda & $4(36,4)$ & & $5(45,5)$ & & $7(63,6)$ & & $6(54,5)$ & & $5(45,5)$ & \\
\hline $\begin{array}{l}\text { Número de } \\
\text { Consultas** }\end{array}$ & & 0,02 & & 0,31 & & 0,40 & & 0,43 & & 0,43 \\
\hline $1-3$ & $10(40,0)$ & & $12(48,0)$ & & $21(84,0)$ & & $15(60,0)$ & & $10(40,0)$ & \\
\hline $4-6$ & $10(58,8)$ & & $12(70,6)$ & & $13(76,5)$ & & $8(47,1)$ & & $9(52,9)$ & \\
\hline$\geq 7$ & $6(100,0)$ & & $4(66,7)$ & & $6(100,0)$ & & $2(33,3)$ & & $4(66,7)$ & \\
\hline $\begin{array}{l}\text { Número de } \\
\text { Gestações }\end{array}$ & & 0,38 & & 0,56 & & 0,52 & & 0,27 & & 0,27 \\
\hline 1 & $22(56,4)$ & & $23(59,0)$ & & $32(82,1)$ & & $19(48,7)$ & & $20(51,3)$ & \\
\hline 2 & $4(44,4)$ & & $5(55,6)$ & & $8(88,9)$ & & $6(66,7)$ & & $3(33,3)$ & \\
\hline
\end{tabular}

*Consultas no Ambulatório de Risco.

Quando analisado o desfecho perinatal das adolescentes com o risco gestacional, aquelas que foram classificadas com condições clínicas pré-existentes apresentaram associação estatística significativa para baixo peso ao nascer $(\mathrm{p}=0,03)$ e as classificadas com alguma intercorrência clínica na atual gestação apresentaram associação estatística significativa para parto pré-termo ( $\mathrm{p}$ $=0,04)$ e baixo peso ao nascer $(\mathrm{p}=0,001)$, conforme apresenta a Tabela 4 .

Tabela 4: Desfecho perinatal de gestantes adolescentes segundo a classificação do risco gestacional em Maringá, PR, Brasil.

\begin{tabular}{lcccccc}
\hline \multicolumn{1}{c}{ Variáveis } & $\begin{array}{c}\text { Condições Clínicas } \\
\text { Pré-existentes } \\
\mathrm{n}(\%)\end{array}$ & $p$ & $\begin{array}{c}\text { Antecedentes } \\
\text { Obstétricos } \\
\mathrm{n}(\%)\end{array}$ & $p$ & $\begin{array}{c}\text { Intercorrências } \\
\text { Clínicas } \\
\mathrm{n}(\%)\end{array}$ & $p$ \\
\hline Pré-Termo (<37 semanas) & $11(61,1)$ & 0,45 & $1(25,0)$ & 0,24 & $9(39,1)$ & 0,04 \\
Baixo peso ao Nascer & $14(77,8)$ & 0,03 & $2(50,0)$ & 0,55 & $8(34,8)$ & 0,001 \\
Apgar 5 minuto $(<7)$ & $14(77,8)$ & 0,33 & $3(75,0)$ & 0,53 & $18(78,3)$ & 0,30 \\
Parto Normal & $9(50,0)$ & 0,82 & $3(75,0)$ & 0,33 & $15(65,2)$ & 0,08 \\
Parto Cesárea & $9(50,0)$ & 0,82 & $1(25,0)$ & 0,33 & $8(34,8)$ & 0,08 \\
\hline
\end{tabular}

\section{DISCUSSÃO}

Os resultados encontrados nessa pesquisa evidenciam que as condições de risco com maior prevalência nas gestantes adolescentes caracterizadas como alto risco são patologias pré-existentes com destaque à hipertensão arterial e à dependência de drogas lícitas/ilícitas e dentre os resultados perinatais desfavoráveis, destaca-se a frequência de consultas no pré-natal de alto risco, o BPN 
relacionado com as condições clínicas pré-existentes e o parto pré-termo e BPN com as intercorrências clínicas na atual gestação.

Em relação à prevalência de hipertensão arterial e dependência de drogas lícitas, corroborando com esses dados, uma revisão sistemática do impacto da idade materna nas complicações clínicas, obstétricas e neonatais na primeira fase da adolescência, publicada em 2014, constatou que adolescentes têm maior prevalência de hipertensão induzida pela gravidez, entre outros fatores, como a eclâmpsia e pré-eclâmpsia, anemia, infecções geniturinárias, hemorragia pós-parto, ruptura de prematura de placenta [11]. A prevalência do consumo de drogas lícitas entre as participantes desta pesquisa se desvela como ponto de atenção, tendo em vista os riscos a que mãe e filho são expostos com esta prática. Em pesquisa realizada com adolescentes estudantes do Estado de Minas Gerais, revelou-se que tal público é carente de informações a respeito do abuso de drogas, sendo que o acesso à informação é retratado como fator de proteção para as mesmas [12]. Em outro estudo, realizado com gestantes adolescentes acompanhadas em uma Unidade Básica de Saúde do Estado do Ceará, constatou-se que embora expressiva maioria $(93,4 \%)$ tenha afirmado conhecer os riscos do consumo de substâncias lícitas durante o período gestacional para o feto, 43,3\% das adolescentes desconheciam os riscos à própria saúde materna [13].

No tocante à frequência de consultas pré-natais, observa-se que $100 \%$ das mães que compareceram a sete ou mais consultas apresentaram parto pré-termo, o que destoa dos achados da literatura. Em estudo realizado com dados de nascimentos dos anos de 2010 a 2012 no município de Piracicaba/SP, observou-se que gestantes com maiores números de consultas pré-natais apresentaram menor incidência de trabalho de parto prematuro comparadas às gestantes com menor número de consultas [14]. Corroborando ainda, outro estudo realizado em 2015 na Bahia, evidenciou que gestantes que realizaram menos de seis consultas pré-natais apresentaram chance 2,94 vezes maior de ter um filho prematuro e com baixo peso ao nascer [15].

No que tange ao número de consultas ideal no pré-natal de gestantes de alto risco, são preconizadas cinco consultas nos centros especializados mais as consultas de pré-natal de risco habitual na atenção primária a saúde, que correspondem a sete consultas no período gestacional e uma no puerpério [10].

Diante desses achados, podemos inferir que o elevado número de consultas se deu devido a adequada assistência ao pré-natal de alto risco, uma vez que, essas gestantes além do fator idade (adolescentes) também apresentavam algum risco de saúde, tornando a gestação de maior risco, necessitando de mais consultas, a fim de evitar outros desfechos desfavoráveis a mãe e ao bebê.

Além disso, é sabido que a prematuridade é um evento de causa multifatorial, porém é possível destacar a própria gravidez na adolescência como um fator de risco para a ocorrência do parto prétermo [16]. Neste estudo, das nove adolescentes que estavam em sua segunda gestação, 44,4\% tiveram parto pré-termo na gestação anterior. Destaca-se que outro fator de risco para o trabalho de parto prematuro é o próprio parto pré-termo enquanto antecedente obstétrico, conforme aponta uma pesquisa realizada na cidade de Lima no Peru, na qual gestantes que apresentavam histórico de trabalho de parto prematuro possuíam risco 3,7 vezes maior para ocorrência de prematuridade na gestação atual, quando comparadas com gestantes que não apresentavam parto pré-termo como antecedente obstétrico [17].

No presente estudo, observou-se que o BPN está relacionado às condições clínicas pré-existentes e, dentre as condições destacadas, estão a hipertensão arterial e a dependência de drogas lícitas. Discordando com nossos achados, um estudo que analisou 2.357 partos de adolescentes, não encontrou associação significativa a hipertensão arterial e tabagismo, analisando-os isoladamente [18]. Vale destacar que a prevalência de gravidez na adolescência por si só já é risco para o BPN [19] e que quando associado a outras patologias poderá desencadear não só o BPN, mas também outros desfechos maternos e perinatais.

Os achados desta pesquisa permitiram identificar que o parto pré-termo e BPN associou-se com as intercorrências clínicas na atual gestação, prevalecendo a má formação fetal e o trabalho de parto prematuro. Corroborando com esses dados, uma pesquisa realizada com 116 puérperas em uma maternidade pública do sul do Maranhão, constatou que o parto pré-termo apresentou associação estatisticamente significante às intercorrências clínicas na gestação $(p=0,001)$, além disso, teve associação com renda mensal inferior a dois salários mínimos $(\mathrm{p}=0,046)$, estresse na gestação ( $\mathrm{p}$ $=0,027)$, primiparidade $(\mathrm{p}=0,044)$ e assistência pré-natal ausente ou inadequada $(\mathrm{p}<0,001)[20]$. 
Em outro estudo, realizado com 3009 puérperas entrevistadas nas capitais e demais municípios da Região Nordeste, 9,7\% apresentaram conceptos com BPN e ao realizar análise bivariada, constatou-se associação significativa entre o BPN e a prematuridade $(\mathrm{p}<0,001)$ [6]. Outro estudo, realizado com 794 gestantes e de seus recém-nascidos constatou que a proporção de baixo peso ao nascer foi de 7,6\% associado principalmente ao fator idade (gestantes menores de 18 anos) [21].

É importante destacar que a gravidez na adolescência ainda está envolvida com aspectos biopsicossociais, sendo forte responsável por abandono ou adiamento dos estudos e dependência financeira dos pais, uma vez que $79,17 \%$ das gestantes observadas não vivem com o companheiro [3]. Uma gestação na adolescência envolve um período de modificações hormonais e psicológicas, podendo comprometer oportunidades futuras de educação e emprego e acarretar danos permanentes à saúde e autocuidado dessa gestante [22].

Como limitação deste estudo, podemos destacar a coleta de dados em fontes secundárias, comprometendo a confiabilidade dos dados, pois dependem da qualidade do preenchimento dos profissionais e a descrição de uma população específica, entretanto, utilizamos uma estratégia de coleta e conferência por dois pesquisadores para evitar algum viés.

\section{CONCLUSÃO}

Fortalecendo as políticas públicas já existentes na saúde materno-infantil e reconhecendo que os desfechos maternos e perinatais dependem de inúmeros fatores, os resultados desse estudo evidenciam que as condições de risco com maior prevalência nas gestantes adolescentes são patologias pregressas com destaque a hipertensão arterial e a dependência de drogas lícitas e dentre os resultados perinatais desfavoráveis, destaca-se o parto pré-termo e o baixo peso ao nascer.

Desta forma, faz-se necessário maior atenção a gestantes adolescentes, e implantação de protocolos multidisciplinares para atendimento em centros especializados, principalmente quando além do fator idade se tem uma outra situação de risco envolvida, como: condições clínicas préexistentes, antecedentes obstétricos e/ou intercorrências clínicas na atual gestação.

\section{REFERÊNCIAS BIBLIOGRÁFICAS}

1. Conceição MA, Vieira ACC, Nunes APN. Gravidez na adolescência, perfil das mulheres que tiveram e não tiveram filho(a). Adolesc Saúde. 2020;17(1):35-40.

2. Sedgh G, Finer LB, Bankole A, Eilers MA, Singh S. Adolescent pregnancy, birth, and abortion rates across countries: levels and recent trends. J Adolesc Health. 2015;56(2):223-230, doi:10.1016/j.jadohealth.2014.09.007

3. Word Health Organization (WHO). Adolescent pregnancy. Genebra. 2018 [citado 2019 ago. 20]. Disponível em: https://www.who.int/en/news-room/fact-sheets/detail/adolescent-pregnancy

4. Verona APA, Dias Júnior CS. Religião e fecundidade entre adolescentes no Brasil. Rev Panam Salud Publica. 2012;31(1):25-31.

5. Ministério da Saúde. Informações sobre Gravidez na Adolescência. 2019. [citado 2019 ago. 30]. Disponível em: http://www.saude.gov.br/saude-para-voce/saude-do-adolescente-e-dojovem/informacoes-sobre-gravidez-na-adolescencia2

6. Almeida AHV, Costa MCO, Gama SGN, Amaral MTR, Vieira GO. Baixo peso ao nascer em adolescentes e adultas jovens na Região Nordeste do Brasil. Rev Bras Saúde Mater Infant. 2014;14(3):279-286, doi: 10.1590/S1519-38292014000300009

7. Gama SGN, Viellas EF, Schilithz AOC, Filha MMT, Carvalho ML, Gomes KRO, Costa MCO, Leal MC. Fatores associados à cesariana entre primíparas adolescentes no Brasil, 2011-2012. Cad Saúde Pública. 2014;30(Suppl. 1):S117-S127, doi: 10.1590/0102-311X00145513

8. Restrepo-Mesa SL, Zapata Lópe N, Parra Sosa BE, Escudero Vásquez LE, Arrovaye LB. Estado nutricional materno y neonatal en un grupo de adolescentes de la ciudad de Medellín. Nutr Hosp. 2015;32(3):1300-1307, doi: 10.3305/nh.2015.32.3.9406

9. Pessoa LS, Saunders C, Belfort GP, Silva LBG, Veras LS, Esteves APVS. Evolução temporal da prevalência de anemia em adolescentes grávidas de uma maternidade pública do Rio de Janeiro. Rev Bras Ginecol Obstet. 2015;37(5):208-215, doi:10.1590/SO100-720320150005321

10. Bouzas ICS, Cader SA, Leão L. Gravidez na adolescência: uma revisão sistemática do impacto da idade materna nas complicações clínicas, obstétricas e neonatais na primeira fase da adolescência. Adolesc Saúde. 2014;11(3):7-21.

11. Paraná. Secretaria Estadual de Saúde. Linha guia: mãe paranaense. Curitiba: SESA, 2018. 63 p. 
12. Santos P, Silva J, Alves M, Rodrigues V. Comportamento de risco à saúde na adolescência: percepção de estudantes de uma escola pública. Rev Multitexto. 2017;4(2): 55-61.

13. Cruz KT, Chaves EMC, Monteiro ARM, Farias LM, Gomes ILV, Dodt RCM. Conhecimentos de adolescents grávidas sobre riscos associados ao uso de substâncias lícitas e ilícitas na gestação. Rev Diálogos Acad. 2013;2(1):41-47.

14. Tuon RA, Ambrosano GMB, Silva SMCV, Pereira AC. Impacto do monitoramento telefônico de gestantes na prevalência da prematuridade e análise dos fatores de risco associados em Piracicaba, São Paulo, Brasil. Cad Saúde Públ. 2016;32(7):e00107014, doi:10.1590/0102-311X00107014

15. Carvalho SS, Coelho JMF, Barcelar DA, Mariola E. Fatores maternos para o nascimento de recémnascidos com baixo peso e prematuros: estudo caso-controle. Ciência \& Saúde. 2016;9(2):76-82.

16. Pérez-Molina J, Panduro-Barón G, Quezada-López C. Factores maternos asociados con nacimiento pretérmino espontáneo versus pretérmino nacido por cesárea. Ginecol Obstet Mex. 2011;79(10):607-612.

17. Ahumada-Barrios ME, Alvarado GF. Factores de Riesgo de parto pretérmino en un hospital. Rev LatinoAm Enferm. 2016;24:e2750, doi: 10.1590/1518-8345.0775.2750

18. Surita FGC, Suarez MBB, Siani S, Silva JLP. Fatores associados ao baixo peso ao nascimento entre adolescentes no Sudeste do Brasil. Rev Bras Ginecol Obstet. 2011;33(10):286-91.

19. Azevedo WF, Diniz MB, Fonseca ESVB, Azevedo LMR, Evangelista CB. Complicações da gravidez na adolescência: revisão sistemática da literatura. Einstein. 2015;13(4):618-626, doi: 10.1590/S167945082015RW3127

20. Almeida AC, Jesus ACP, Lima PFT, Araújo MFM, Araújo TM. Fatores de risco maternos para prematuridade em uma maternidade pública de Imperatriz-MA. Rev Gaúcha Enferm. 2012;33(2):86-94.

21. Moreira AI, Sousa PR, Sarno F. Baixo peso ao nascer e seus fatores associados. Einstein. 2018;16(4):16, doi: 10.31744/einstein_journal/2018AO4251

22. Sousa EZT, Sousa CAS, Guimarães FM, Barroso ID, Sousa KLS, Gomes MC, Gonçalves SR. Qualidade de vida de adolescentes grávidas. Rev Eletr Acervo Saúde. 2020;12(6):e3161, doi: 10.25248/reas.e3161.2020 